\title{
Multiple guide star tomography demonstration at Palomar Observatory
}

V. Velur, R. C. Flicker, B. C. Platt, M. C. Britton, R. G. Dekany, et al.

V. Velur, R. C. Flicker, B. C. Platt, M. C. Britton, R. G. Dekany, M. Troy, J. E. Roberts, J. C. Shelton, J. Hickey, "Multiple guide star tomography demonstration at Palomar Observatory," Proc. SPIE 6272, Advances in Adaptive Optics II, 62725C (27 June 2006); doi: 10.1117/12.671666

Event: SPIE Astronomical Telescopes + Instrumentation, 2006, Orlando, Florida, United States 


\title{
Multiple guide star tomography demonstration at Palomar observatory
}

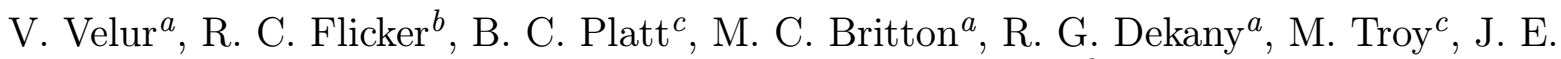

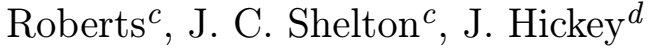 \\ ${ }^{a}$ California Institute of Technology, Pasadena, CA 91125, USA; \\ ${ }^{b}$ W. M. Keck Observatory, Kamuela, HI 96743, USA; \\ ${ }^{c}$ Jet Propulsion Laboratory, 4800 Oak Grove Drive, Pasadena, CA 91109, USA \\ ${ }^{d}$ Palomar Observatory, 35899 Canfield Rd., Palomar Mountain, CA 92060-0200, USA.
}

\begin{abstract}
We have built and field tested a multiple guide star tomograph with four Shack-Hartmann wavefront sensors. We predict the wavefront on the fourth sensor channel estimated using wavefront information from the other three channels using synchronously recorded data. This system helps in the design of wavefront sensors for future extremely large telescopes that will use multi conjugate adaptive optics and multi object adaptive optics. Different wavefront prediction algorithms are being tested with the data obtained. We describe the system, its current capabilities and some preliminary results.
\end{abstract}

Keywords: Multi-Conjugate adaptive optics, tomography, novel wavefront sensing

\section{INTRODUCTION}

The Palomar tomograph (PT) is a compact multiple guide star wavefront sensor system that can be used to test different tomographic wavefront sensing algorithms. ${ }^{1}$ The implementation and performance characterization of these algorithms will be the driving force for wide field adaptive optics (AO) and in turn AO fed spectroscopy. This is a key technology development effort for the next generation of AO systems on 8-10m class telescopes and for the planned Thirty Meter Telescope (TMT) and the Giant Magellan Telescope (GMT) projects ${ }^{3} 65.8$ Using $\mathrm{PT}$ we are comparing different algorithms and assessing the importance of a priori turbulence statistics. Slope detection and ranging (SLODAR) experiments to determine the heights of strong turbulence layers are also being performed using cross-correlation of wavefront sensor data obtained. ${ }^{2}$ Palomar currently has a multi-aperture scintillation sensor and a differential image motion monitor (MASS and DIMM unit), on loan from the TMT project office, which enables us to monitor and characterize the atmospheric turbulence concurrent with our PT observations.

\section{SYSTEM DESCRIPTION:}

Five low noise CCD based Shack Hartmann wavefront sensors have been integrated into the existing the Palomar Adaptive Optics (PALAO) bench. The five wavefront sensors consist of three 16x16 sub-aperture channels and one $3 \times 3$ sub-aperture low order wavefront sensor (LOWFS) inside the PT enclosure and one 16x16 Active High Order Wavefront Sensor (AHOWFS) outside the enclosure that receives light from a small reflective field stop. The three 16x16 wavefront sensors inside the enclosure read out $64 \times 64$ pixels with $4 \times 4$ pixels/sub-aperture while the AHOWFS reads out $64 \times 64$ pixels that are binned on chip to provide $32 \times 32$ active super-pixels. The LOWFS has $27 \times 27$ active pixels that are binned $3 \times 3$ to provide $9 \times 9$ active super pixels. The four wavefront sensor (WFS) channels inside the PT enclosure can traverse an almost continuous field of $\sim 90$ arc-seconds, while a pair of mirrors in PALAO can be moved so that the active wavefront sensor can acquire guide stars. Between these two acquisition systems, we can acquire and guide on asterisms where the maximum separation between stars is less than 90 arc-sec.

Author to whom correspondence should be sent: V. Velur - Robinson 01, Mail Code - 105-24 Caltech, 1200 E California Blvd., Pasadena, CA - 91125. E-mail: vnv@phobos.caltech.edu

Advances in Adaptive Optics II, edited by Brent L. Ellerbroek, Domenico Bonaccini Calia, Proc. of SPIE Vol. 6272, 62725C, (2006) - 0277-786X/06/\$15 - doi: 10.1117/12.671666 


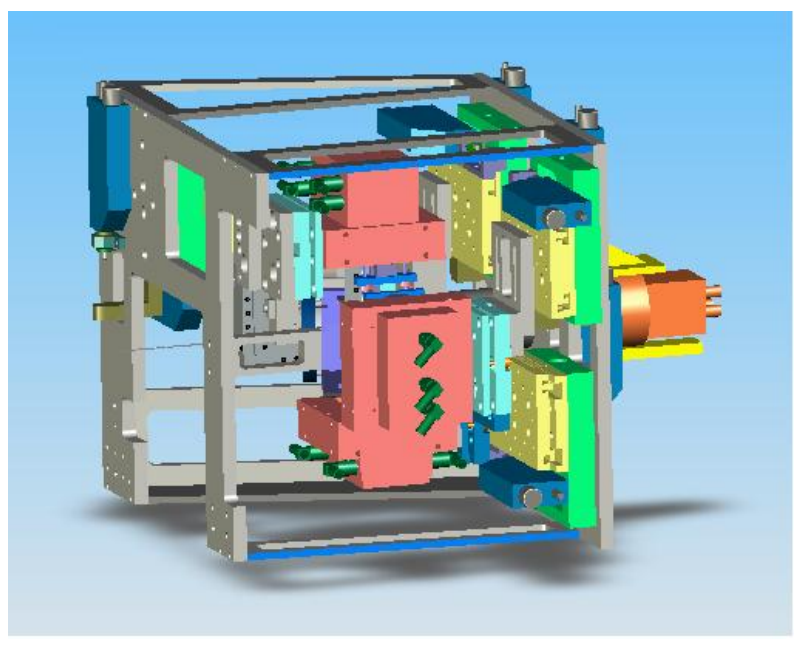

Figure 1. Three dimensional rendering of the multiple guide star tomograph showing 4 MGSU channels. Each WFS is mounted on a tip-tilt stage for accurate alignment. The tip-tilt stage itself is mounted on $\mathrm{X}$ and $\mathrm{Y}$ translation stages for picking off stars in the FoV.

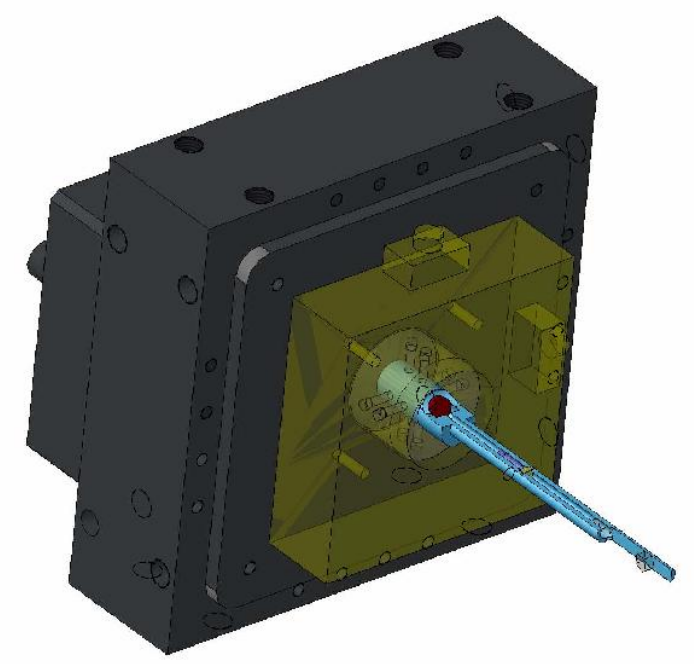

Figure 3. Depiction of a single channel of the MGSU unit with penta prism pick-off, collimator, lenslet array, relay optics and detector.

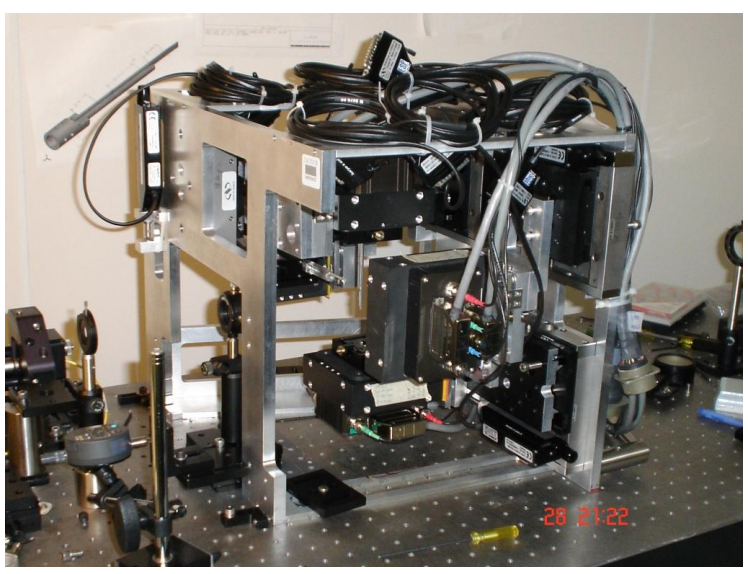

Figure 2. The Tomograph as built in the lab.

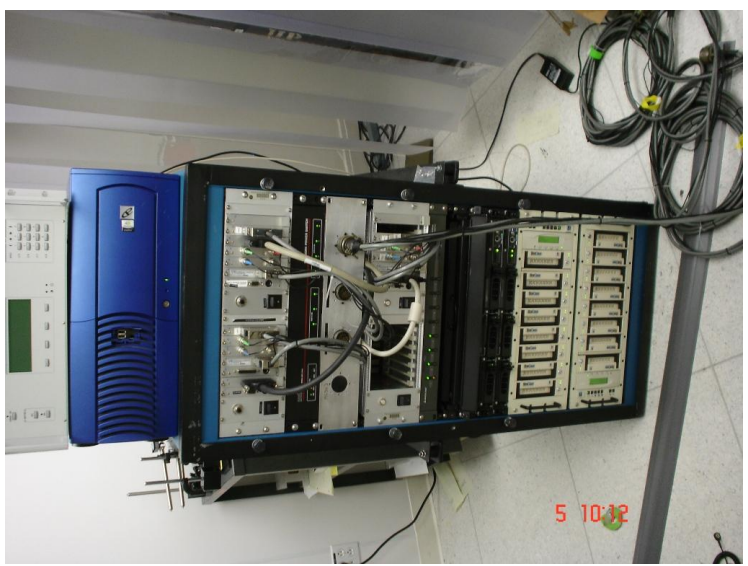

Figure 4. Shows the PT electronics rack with the 8 channel motion controller, a Windows test PC, camera controller, network power switch, four $1 \mathrm{U}$ Dell PCs and two RAID disks. 


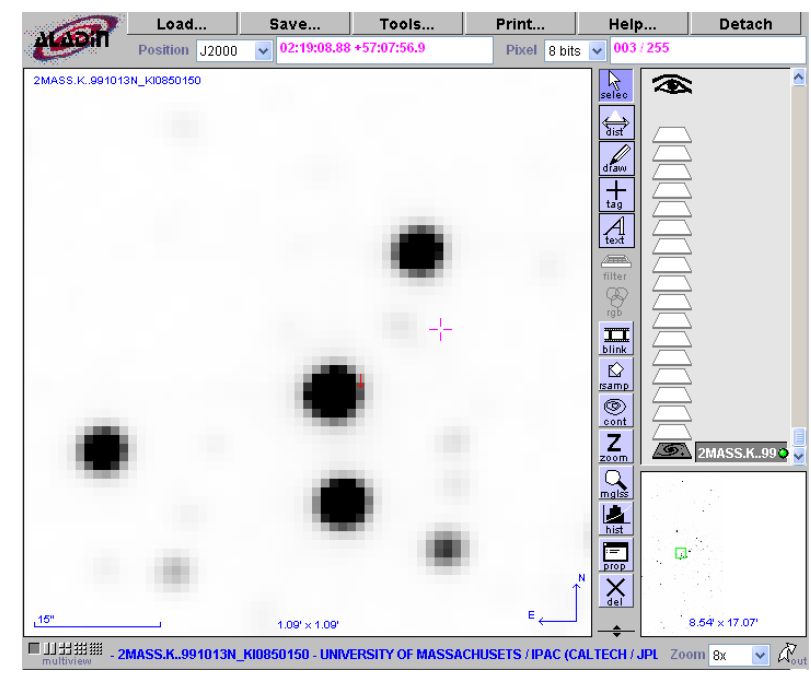

Figure 5. Asterism in Aquila (SAO23181) used for our tests. WFS data from the 3 bright stars was used to predict the wavefront from the central brightest star. The truth sensor was the PALAO high order WFS which was also used to close the tip-tilt loop of the AO system.

All five wavefront sensors use cameras and controllers made by SciMeasure Analytical Systems, Inc. (SAS) that grab frames at a maximum rate of $2000 \mathrm{~Hz}$ when running in $64 \mathrm{x} 64$ pixel read out mode. These cameras have a measured $3 e^{-} s / \mathrm{sec}$ read noise at $500 \mathrm{~Hz}$ and the noise level creeps up to $7 e^{-} s / \mathrm{sec}$ at $2000 \mathrm{~Hz}$. The LOWFS serves as our tip, tilt, focus and astigmatism sensor for Laser Guide Star (LGS) operations and does not play a part in the tests described.

PT's pick off arms can traverse an almost continous field of 90 arc-sec. diameter to aquire guide stars. The optical train is designed to be telecentric over this range to keep pupil shear to within $1.2 \%$ at the lenslet pupil over this field of view. The system can be operated with PALAO's tip-tilt and high order AO loop closed, with both open or with just the tip-tilt loop closed. A custom timing module is used to trigger the wavefront sensing channels to run at integral frame rates of a master trigger as shown in Figure 6. This facilitates use of the system with guide stars of different magnitudes. Different program selections are available on the SAS cameras to enable data to be recorded at frame rates between 50-2000 Hz. The $14 \mathrm{bit}, 64 \mathrm{x} 64$ pixel data from the the channels inside the enclosure and 14 bit $32 \times 32$ on-chip binned pixel data from the AHOWFS are recorded onto two striped RAID disks with 3.2 Terabytes of storage space.

\section{SIMULATION AND DATA ANALYSIS ALGORITHM DEVELOPMENT}

\subsection{Description of the experiment}

The general scope of the experiment is to use the information from three natural guide stars (NGS) to estimate the wavefront at a fourth position, where a fourth NGS is available to provide a truth measurement. Performance is assessed by comparing the truth measurement against the prediction from the three NGSs. The simplest asterism for this type of estimation and validation would be to have three stars at the vertices of an equilateral triangle that feed the tomography sensors and a fourth star at the centroid serving as the truth sensor. We use the AHOWFS of the PALAO system on the central star to act as truth sensor, and the three MGSU cameras on the surrounding stars act as the tomographic WFSs. A variation of this experiment may be carried out using only three NGSs, where two are employed to make a prediction for the third, which may be located off-axis rather than in the central region. This variant of the original experiment mimics the situation in certain MOAO (multi-object adaptive optics) designs or to sharpen an off-axis tip-tilt NGS based on on-axis LGS tomography.

Given simultaneous measurements from four WFSs on a 4-star asterism as described above, one can apply a number of different analysis methods that will answer a variety of questions about the data set and the outcome 


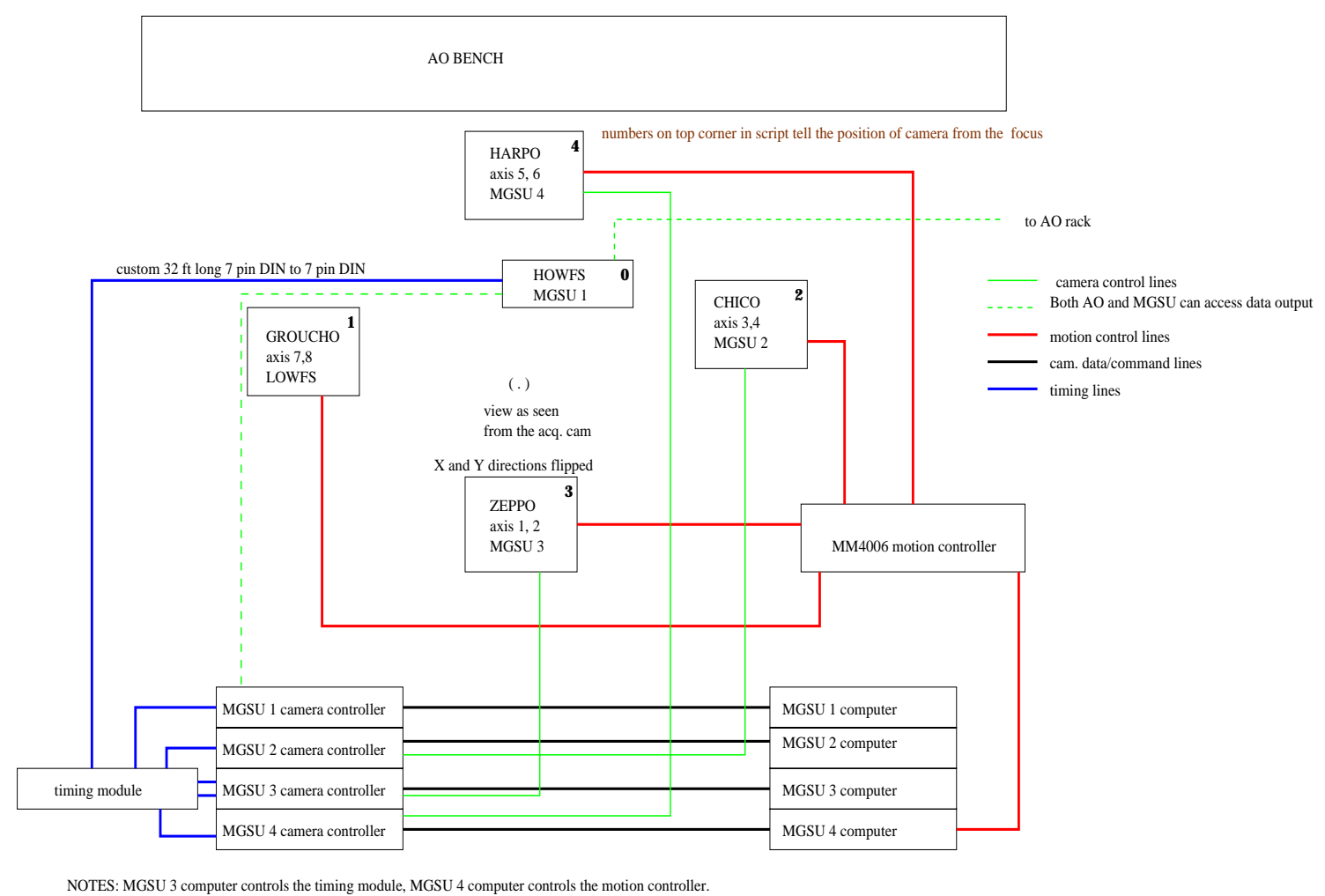

Figure 6. Schematic of the MGSU/PT showing the data, control and timing lines

of the experiment. The analysis methods that we are considering for the MGSU data analysis include:

- Wavefront reconstruction from centroids,

- Wallner-type ${ }^{4}$ statistical estimator on centroids,

- Zernike mode reconstruction and spatial correlation analysis,

- Cross-validation of tomography estimation results by simulation

\section{FIELD TESTS}

During the nights of 9 and 10th of February, 2006, we observed a 4-star asterism with the PT in moderate seeing conditions. As part of our preparation for the observing run, we isolated a few asterisms with the preferred pattern described previously. We then downsized the list to those we could observe in the time window allotted to us. We rotated the telescope Cassegrain ring so that we could acquire all four stars, as there is a small dead zone of a few seconds of arc around each guide star due to the finite size of the pick off prism and its mounting structure. An asymmetric actuator poke pattern was applied on the deformable mirror to determine the orientation of each detector with respect to the incoming light. A special build of the PALAO software was used to allow the AHOWFS to be triggered externally from our timing module that triggered all four cameras. The Sterhl was maximized by using PALAO's standard flat-field tuning procedure. Two data output cards were used on the AHOWFS camera controller so that it could feed both the AO real time computer and the MGSU data recording system at the same time. With the two cards we could lock the tip-tilt and high order DM loop individually or simultaneously. We pointed the telescope to the star field shown in figure 5, acquired the brightest central star on the AHOWFS and locked the tip-tilt loop. The other three stars were acquired so that the pupils 


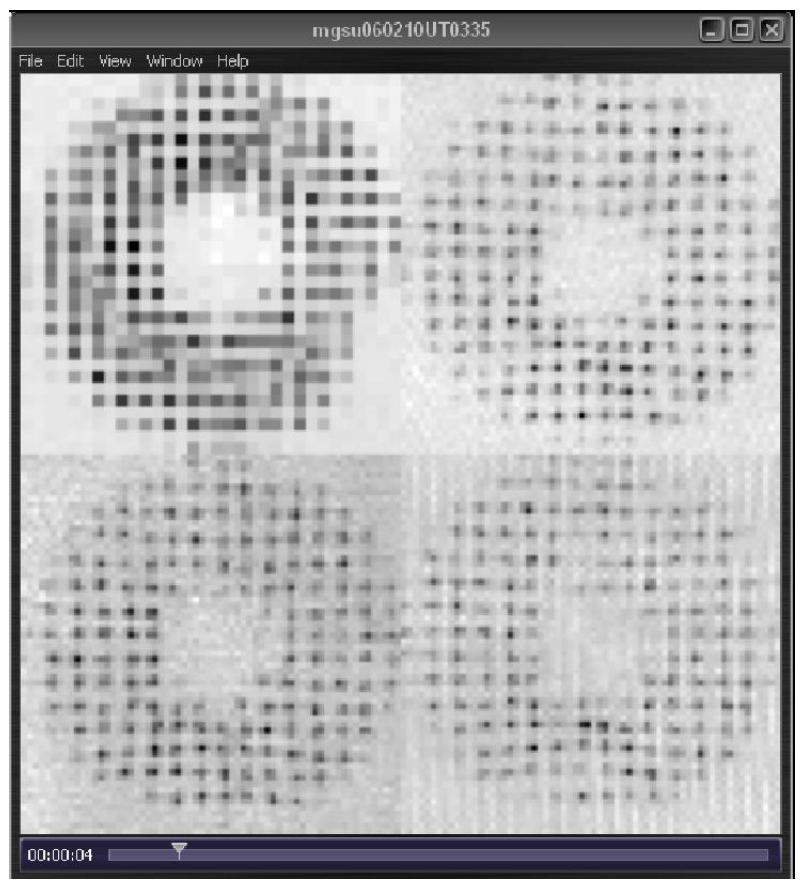

Figure 7. Image showing a single frame of on-sky data from each of the four WFS channels. In this case, the tip-tilt loop was locked using the high order wavefront sensor ( $32 \times 32$ sub-aperture), and the other 3 cameras were recording data in $64 \times 64$ mode.

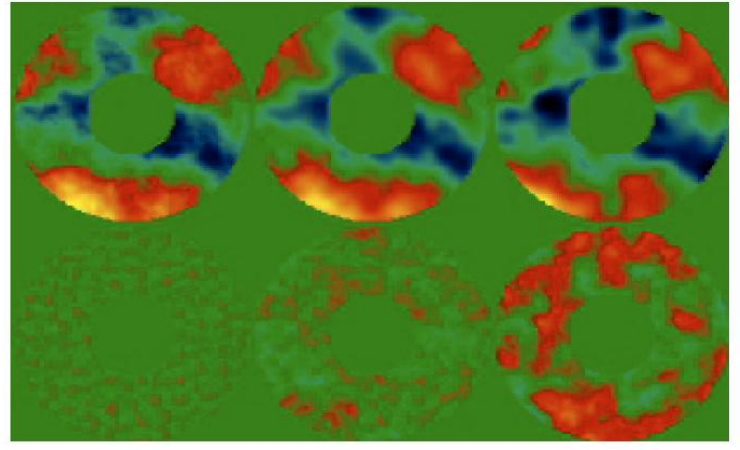

Figure 8. Sample simulation result screen from main IDL analysis code. Top row - wavefronts: pure open loop turbulence (left); HOWFS reconstructed wavefront (center), and MGSU reconstructed wavefront (right). Bottom row - residual wavefront errors w.r.t. the true wavefront: optimal fit of $17 \times 17$ actuator DM (left); HOWFS reconstructed wavefront error (center); MGSU reconstructed wavefront error (right).

were centered and the sub-apertures aligned. Then we unlocked the tip-tilt loop, and began the data acquisition. After a few seconds of data acquisition the tip-tilt loop was locked to keep the images on each of the detectors stable. The DM loop was locked for the last minute of the exposure. Data was recorded at a few different frame rates ranging from 60 to $256 \mathrm{~Hz}$ (limited by guide star magnitude). Figure 9 shows our observation log and includes information about the asterism we observed. A sample frame from this data taken is shown in figure 7 .

\subsection{Analysis implementation and preliminary results}

The data analysis is distributed over various existing codes and additional code developed for this experiment. The wavefront reconstruction matrices were generated using the Yorick Adaptive Optics (YAO) simulation code ${ }^{7}$ modified specifically for MGSU simulations. Figure 12 shows sample initial results for estimating the wavefront $w$ from the vector $v$ of MGSU measurement, obtained through the following linear estimation steps:

$$
\begin{aligned}
w & =H G^{+} \widehat{u}, \\
\widehat{u} & =E v,
\end{aligned}
$$

where $G^{+}$is the pseudo-inverse of a simulated interaction matrix for the AHOWFS, and $H$ is a set of influence functions. Both were modeled in YAO. $E$ is the statistical least-squares tomographic estimator:

$$
E=\left\langle u v^{t}\right\rangle\left\langle v v^{t}\right\rangle^{-1}
$$

where $u$ is the on-axis (AHOWFS) centroid vector. Figure 8 shows a sample frame from a YAO simulation that was completed prior to the on-sky experiment in order to asses what we may expect from the real data. 


\begin{tabular}{|c|c|c|c|c|c|}
\hline Experiment \# & \multicolumn{2}{|l|}{3} & Asterism \# & \multicolumn{2}{|c|}{ A1 } \\
\hline Date/time & \multicolumn{2}{|l|}{$2006-02-10$} & RA (ป2000) & \multicolumn{2}{|c|}{$02: 19: 10$} \\
\hline UT & \multicolumn{2}{|l|}{$03: 35$} & DEC (ป2000) & \multicolumn{2}{|c|}{$+57: 07: 50$} \\
\hline FSM loop & \multicolumn{2}{|l|}{ closed } & ZD/airm & \multicolumn{2}{|c|}{1.27 airm } \\
\hline \multirow[t]{2}{*}{ DM loop } & \multicolumn{2}{|l|}{ open } & Cass ring & \multicolumn{2}{|c|}{205.32 deg } \\
\hline & HOWFS & $\begin{array}{c}\text { HGSU } 2 \\
\text { (Chico) }\end{array}$ & $\begin{array}{l}\text { MGSU } \\
\text { (Zерpo }\end{array}$ & & $\begin{array}{l}\text { HGSU } 4 \\
\text { (Harpo) }\end{array}$ \\
\hline format & $32 \times 32$ & $64 \times 64$ & $64 \times 64$ & & $64 \times 64$ \\
\hline program/cfg. & 5/Lil-Joe-32×32 & 5/Lil-Joe-64 & 5/Lil-Joe & & 5月il-Joe-64 \\
\hline d. frame rate & 256 & 256 & 256 & & 256 \\
\hline gain & 2 & 0 & 0 & & 0 \\
\hline star name & SAO 23181 & $\mathrm{BD}+56526$ & $\mathrm{BD}+565$ & & $\mathrm{BD}+56525$ \\
\hline magnitude & $8.43 \mathrm{~V}$ & $8.98 \mathrm{~V}$ & 10.21 & & $9.24 \mathrm{~V}$ \\
\hline exposure time & 308.075 & & & & \\
\hline frame rate & 256.064 & & & & \\
\hline \# frames & 78887 & & & & \\
\hline
\end{tabular}

Figure 9. Observing log showing the star brightnesses and camera speeds for one data set.
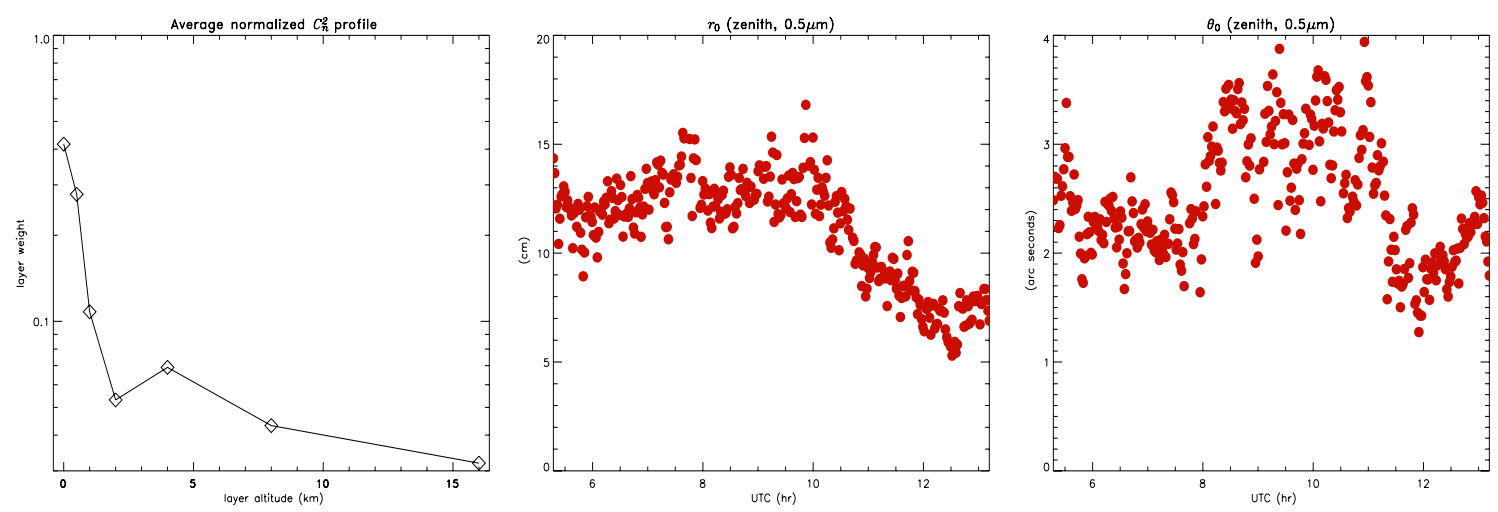

Figure 10. The top left image shows the $C_{n}^{2}$ profile averaged over the night for 7 layers of the atmosphere. Center and right images show the time evolution of the isoplanatic angle and the Fried parameter.

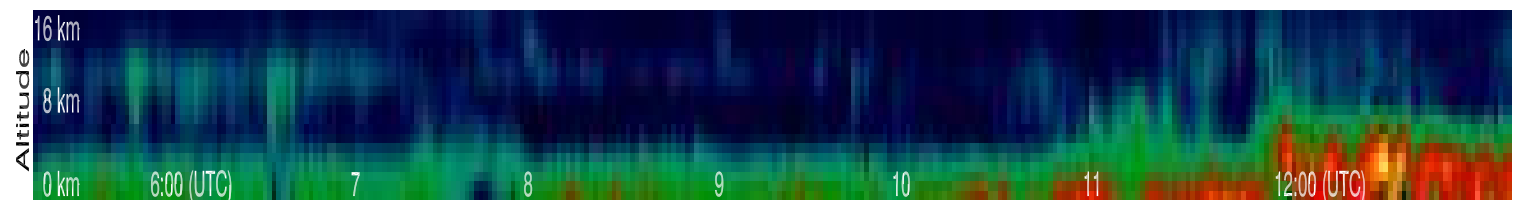

Figure 11. Time evolution of $C_{n}^{2}$ 


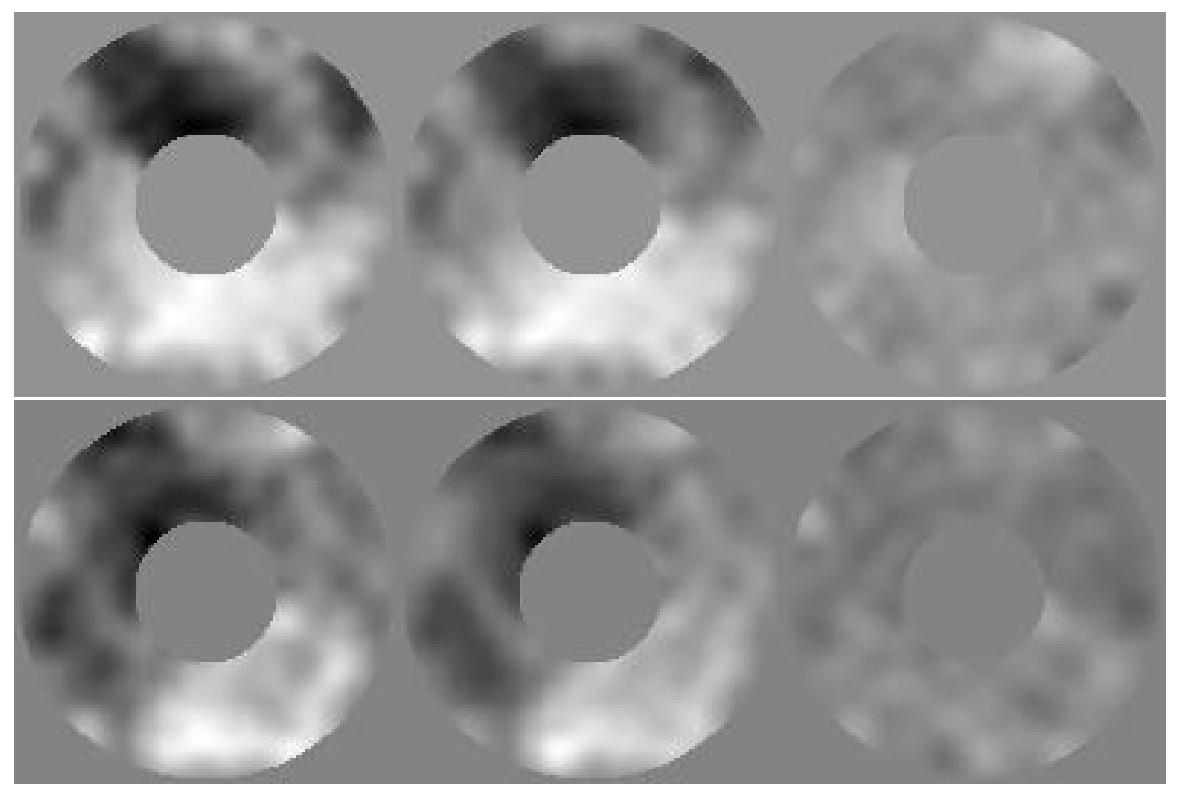

Figure 12. Two sample analysis frames from the 10 February 2006 data set, with the tip-tilt loop closed on the HOWFS. Left column: wavefronts reconstructed directly from HOWFS (truth wavefront). Center column: wavefronts estimated from MGSU measurements. Right column: residual wavefronts.

Figure 12 shows a sample analysis frame from the data set taken on 10 February 2006. The value of $r_{0}$ and $\theta_{0}$ were obtained from the MASS DIMM measurements. The variation of $r_{0}$ and $\theta_{0}$ during the night is shown in Figures 10 and 11.

We estimated a Fried parameter of $r_{0} \approx 10 \mathrm{~cm}$ from the MASS/DIMM unit during the above five minute exposure. Kolmogorov theory suggests that a $r_{0}=10$ should produce a tip/tilt-removed RMS wavefront error of $759 \mathrm{~nm}$ over a $5-\mathrm{m}$ aperture. The central obscuration of the Hale telescope and the high-spatial frequency cut-off of the AHOWFS (estimated at $1 / 31.2 \mathrm{~cm}^{-1}$ ) lowers the Kolmogorov predicted RMS wavefront error to $671 \mathrm{~nm}$.

From the wavefronts reconstructed directly from the AHOWFS (left column of Figure 12), we estimate for this data set an RMS wavefront error of $598 \mathrm{~nm}$ (left column of Figure 12). Figure 13 shows that most of the discrepancy is in the low-order modes, which in our estimation has a lower variance the Kolmogorov statistics would predict. Using 40,000 frames of this data set, we measure an RMS error in the tomographic wavefront estimation of $231 \mathrm{~nm}$ (right column of Figure 12), when using the statistical least-squares estimator in equations (1)-(3). Currently, our results from this preliminary estimate are limited by the following:

- Noise in the WFS camera that was gathering data from the faintest star.

- Internal calibration errors, pupil registration, telecentricity (we have not yet measured the actual pupil shear across the pupil) and cross talk between sub-apertures.

- The use of the statistical least-squares tomographic estimator, which may be significantly non-optimal. Modeled maximum a posteriori estimators are being constructed and will be applied to the data sets in the next phase of the analysis.

A more rigorous analysis is underway to quantify the errors in our tomographic estimation. 


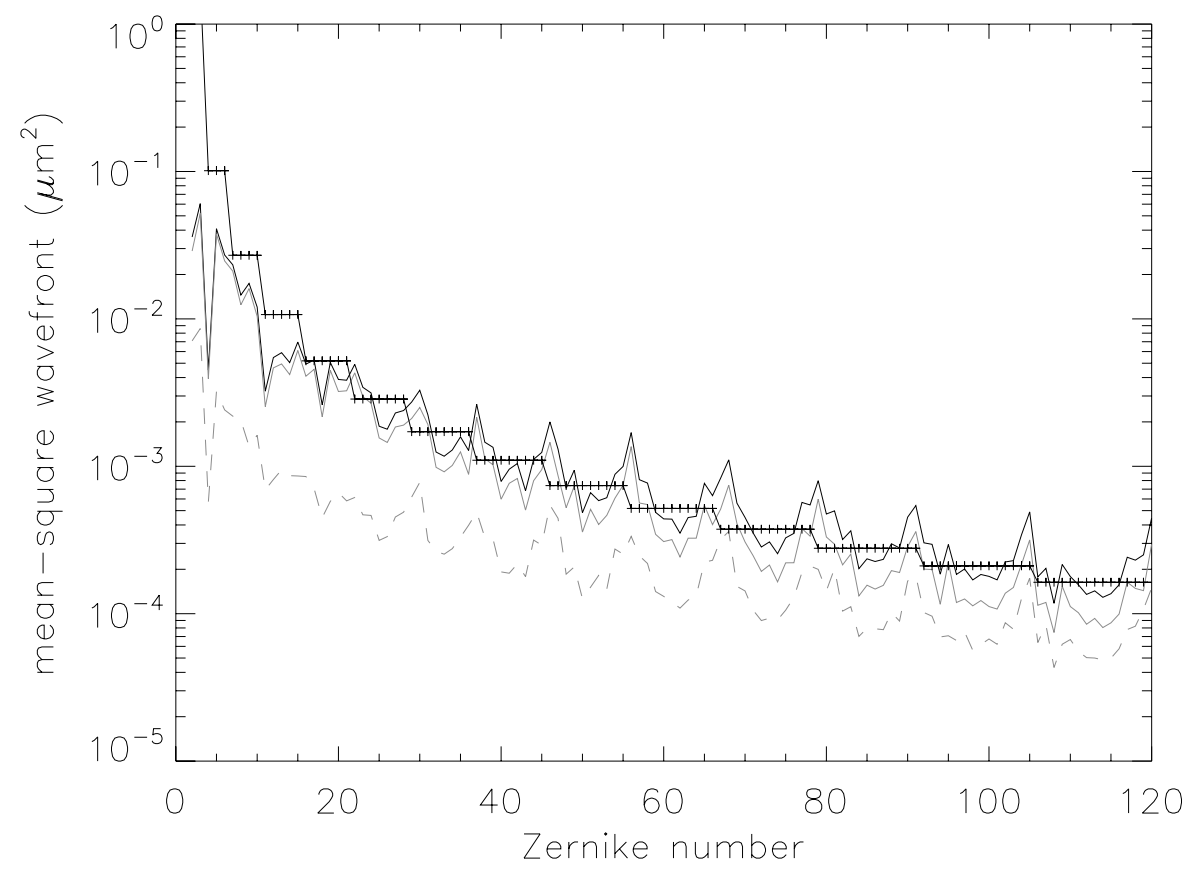

Figure 13. Zernike coefficients from estimated wavefronts (fitted with a central obscuration). Plotted are the estimated RMS wavefront errors for the HOWFS direct reconstruction (solid black line), tomographic estimation (solid grey line), and the residual error (dashed grey line). For comparison is also plotted the theoretical Zernike variances for an unobscured aperture (solid black line and pluses).

\section{CONCLUSION}

We built and field tested a four channel tomograph unit. We estimated the wavefront error of the central star from the three surrounding ones to $231 \mathrm{~nm}$ RMS error using a Wallner-type estimator.

We developed a scheme to align and manufacture wavefront sensors in a batch process using special jigs that give us an understanding of how to replicate wavefront sensors for use in wide-field AO for ELTs. With more field-testing of the current system we will learn to calibrate the it better and gain knowledge on how to acquire stars quickly. This will greatly improve the observing efficiencies of large observatories and is also an issue with most single laser guide star systems.

\subsection{Future Work}

After gaining confidence in our tomography algorithms and optimizing them, the experiment can be refined to obtain better estimates of the wavefront at the truth sensor.

The tomographic reconstruction currently works in open loop (done in post-processing), but the system was designed with the intention of operating in closed loop with a real-time computer that drives the existing DM using wavefront information obtained from all WFSs. The ELTs require tomographic WFSs operating in real time.

The Caltech, JPL and The University of Chicago PALAO team is currently planning a major upgrade of existing Laser Guide Star (LGS) AO system. Firstly, we are upgrading our 241 active element AO system to a 3000 active element system. ${ }^{9}$ As a second step we are upgrading the laser in our LGS system from a $8.5 \mathrm{~W}$ to a $20 \mathrm{~W}$ system. Lastly, an optical integral field spectrograph designed to take advantage to the nearly diffraction limited Point Spread Functions (PSFs) produced by PALAO called Oxford Short Wavelength Integral 
Field Spectrograph (OSWIFT) is being built by Oxford University, U.K. to go behind the PALAO. A variety of experiments and use for the MGSU can be devised, keeping in mind, the grand scheme of PALAO upgrades planned in the imminent future.

\section{ACKNOWLEDGMENTS}

This work was supported by the National Science Foundation (NSF) under Agreement No. AST-0096928, the Thirty Meter Telescope (TMT) project, W. M. Keck Observatory and internal Caltech Optical Observatories funds. The authors thank the JPL AO team and staff of Palomar Observatory for valuable support rendered at various stages of this project. Special thanks to Prof. Ed Kibblewhite (The University of Chicago) for useful insight during the design phase. We would also like to thank the TMT site-selection team and Dr. A. J. Pickles (COO) for ensuring that the MASS/DIMM unit was up and working during these experiments. In particular, the efforts of Reed Riddle, Warren Skidmore, Matthias Schöeck, Sebastian Els (CTIO) and Tony Travouillon to keep the unit in perfect running condition despite their busy TMT site survey activities.

The Thirty Meter Telescope (TMT) Project is a partnership of the Association of Universities for Research in Astronomy (AURA), the National Science Foundation (NSF), the Association of Canadian Universities for Research in Astronomy (ACURA), the California Institute of Technology and the University of California. The partners gratefully acknowledge the support of the Gordon and Betty Moore Foundation, the US National Science Foundation, the National Research Council of Canada, the Natural Sciences and Engineering Research Council of Canada, and the Gemini Partnership.

\section{REFERENCES}

1. R.Dekany, M. Troy, K. Wallace, C. Bleau, R. DuVarney, G. Motter, Multiple Guide Star Wavefront Sensing for Palomar AO, Beyond Conventional Adaptive Optics, Venice, Italy 2001.

2. L. Wang, University of California, Irvine, CA, Private communication.

3. R. Ragazzoni, E. Marchetti and G. Valente, Adaptive optics correction for the whole sky, Nature 403, pp 54-56

4. E.P. Wallner, Optimal wave-front correction using slope measurements, JOSA-A, 73, Dec. 1983, pp 1771-1776.

5. D. Gavel, Tomography for multiconjugate adaptive optics systems usinglaser guide stars, Vol. 5490, SPIE Glassgow 2004.

6. P. Knutsson and M. Owner-Petersen, Emulation of dual-conjugate adaptive optics on an 8-m class telescope, Opt. Express, 11, 2231-2237 (2003).

7. F. Rigaut, http://www.maumae.net/yao/aosimul.html

8. C. Baranec, B. Bauman M. Lloyd-Hart, Concept for a laser guide beacon Shack-Hartmann wave-front sensor with dynamically steered subapertures, Optics Letters, 30, 693-695, 2005.

9. R. G. Dekany, A. H. Bouchez, M. C. Britton, V. Velur, PALM-3000: visible light AO on the 5.1-m Telescope, submitted to SPIE Orlando 2006. 